\title{
Persistence to disease-modifying therapies for multiple sclerosis in a Canadian cohort
}

\author{
This article was published in the following Dove Press journal: \\ Patient Preference and Adherence \\ 28 June 2017 \\ Number of times this article has been viewed
}

\section{Dessalegn Y Melesse ${ }^{1,2}$ \\ Ruth Ann Marrie ${ }^{2,3}$ \\ James F Blanchard' \\ Bo Nancy $\mathrm{Yu}^{1,4}$ \\ Charity Evans ${ }^{5}$}

'Centre for Global Public Health, ${ }^{2}$ Department of Community Health Sciences, ${ }^{3}$ Department of Internal Medicine, University of Manitoba, ${ }^{4}$ Public Health, Manitoba Health Seniors and Active Living, Winnipeg, Manitoba, ${ }^{5}$ College of Pharmacy \& Nutrition, University of Saskatchewan, Saskatoon, Saskatchewan, Canada
Correspondence: Charity Evans College of Pharmacy \& Nutrition, University of Saskatchewan, 104 Clinic Place, Saskatoon, SK, S7N 2Z4, Canada

Tel +l 3069662836

Fax +I 3069666377

Email charity.evans@usask.ca
Purpose: To examine the long-term persistence to the first-line injectable disease-modifying therapies (DMTs) for multiple sclerosis (MS) and to identify the factors associated with nonpersistence.

Patients and methods: We used population-based administrative data from Manitoba, Canada. All adult subjects who were diagnosed with MS and dispensed a first-line injectable DMT (beta-interferon-1b, beta-interferon-1a, and glatiramer acetate) between 1996 and 2011 and had a minimum of 1 year of follow-up were included. The primary outcome was the median time to discontinuation of any DMT. The associations between potential predictors and persistence were estimated using multivariable Cox-proportional hazard models.

Results: Overall, 721 subjects were followed for a median of 7.8 years (interquartile range 6.1). The median time to discontinuation of all first-line DMTs was 4.2 years (25th and 75 th percentile: $1.7,10.6$ years). Of the $451(62.6 \%)$ subjects who discontinued their DMT during the study period, 259 (57.4\%) eventually resumed or restarted a DMT. Subjects who were younger when starting a DMT, had prior MS-related hospitalizations, were more recently diagnosed with MS, or had a greater lag time between their MS diagnosis and DMT initiation were more likely to discontinue therapy.

Conclusion: Over half of the individuals receiving a DMT for MS in Manitoba remained on therapy for at least 4 years. DMT discontinuation occurred in $60 \%$ of the cohort, but most restarted a DMT within 1 year. While not all of the factors identified with discontinuing DMT are modifiable, they may help practitioners enhance MS care by identifying individuals who may be at particular risk for DMT discontinuation.

Keywords: drug utilization, discontinuation, adherence, administrative data

\section{Introduction}

In the past two decades, several disease-modifying therapies (DMTs) have become available for the treatment of relapsing-onset multiple sclerosis (MS). While all DMTs reduce disease activity to some extent, the efficacy rates vary. ${ }^{1-3}$ However, the success of any pharmacological management for MS relies on the adherence and persistence to the prescribed therapy; even the most potent DMT will have little benefit if the drug is not taken, or not taken correctly.

Adherence is typically reported as the proportion of doses taken as prescribed over a specific time period, while persistence is the length of time on the drug. ${ }^{4,5}$ Poor adherence and persistence have been associated with increased morbidity, mortality, and healthcare costs in several chronic conditions, ${ }^{6-9}$ and there is some evidence to suggest the same is true in MS. ${ }^{10-12}$

Studies examining adherence and persistence to the injectable DMTs for MS have reported varying results. Good adherence, usually defined as taking $\geq 80 \%$ of doses 
correctly, ranges from $40 \%$ to $88 \%, 5,13,14$ and the reported proportions of nonpersistence vary from $9 \%$ within 6 months to $41 \%$ within 3 years. ${ }^{14,15}$ These differences are likely due to inconsistencies in the study follow-up time, definitions and measures of persistence, and cohort selection, ${ }^{16-22}$ making it difficult to compare results across studies or to delineate those individuals who may be at risk for poor adherence or persistence. Identifying patients at risk for nonpersistence is important for developing strategies aimed at supporting appropriate DMT use. Therefore, we aimed to characterize the long-term persistence patterns to the first-line injectable DMTs (betainterferons and glatiramer acetate) and to identify factors associated with nonpersistence over a 15-year period, using population-based data from Manitoba, Canada.

\section{Methods}

\section{Data source}

We used population-based health administrative data from Manitoba, a central Canadian province with a relatively stable population of 1.27 million. Manitoba Health, Seniors and Active Living is a publicly funded health insurance agency providing comprehensive health insurance to nearly all $(98 \%)$ residents in the province. ${ }^{23}$ It maintains administrative databases which provide information on the registered population, and health services used, including physician billing and hospitalizations since 1984. Since 1995, the Drug Program Information Network database has captured information on all prescription drugs dispensed in an outpatient setting, including the drug identification number (DIN), the dispensation dates, the quantity dispensed, and the days supplied. For this study, we used hospitalization, physician billing, and prescription claims data which were linked using an anonymized unique personal identifier.

\section{Study cohort}

All incident cases of MS diagnosed between April 1, 1996, and March 31, 2011, were identified using a validated definition requiring $\geq 3$ claims for MS based on any combination of physician, hospital, or prescription claims. ${ }^{24}$ Physician and hospital claims were identified using the International Classification of Disease (ICD)-9 or -10 codes (Table S1), and prescription claims were limited to those for DMTs for MS. We considered the date of the first claim for a demyelinating disease of the central nervous system, including MS, as the date of diagnosis. ${ }^{24}$ Those subjects who were first dispensed a DMT for relapsing-onset MS (beta-interferon-1b, beta-interferon-1a, and glatiramer acetate) during this time period, were $\geq 18$ years of age, and had a minimum of 1 year of follow-up after their first DMT dispensation (index date) were included in the study. Because the first DMT was approved in Canada in mid-1995, but did not receive provincial formulary coverage in Manitoba for another 2 years, virtually all included subjects were incident, or "new," users. Natalizumab and oral DMTs were neither licensed nor commonly used over the study period and therefore could not be assessed or reported because of privacy protocols. The subjects were followed from their index date until their exit date: death, cancellation of health insurance coverage (eg, emigration), or study end (March 31, 2012), whichever came first.

\section{Study outcomes}

The primary outcome was the median time to discontinuation of any DMT from the index date (ie, persistence). A discontinuation of any DMT was determined by a $>90$-day gap in therapy, and the discontinuation date was set as the date of the last dispensation plus the number of days of drug supplied in the last dispensation. A 90-day gap was chosen to avoid misclassifying subjects who were switching between therapies and to be consistent with previous studies examining persistence in MS. ${ }^{14,25,26}$ Because women are advised to discontinue DMT 3 months before conception, women with a delivery diagnosis code (ICD-9/10 codes of "630-679" and "O00-O99") were censored 1 year before their delivery date, or at their discontinuation date if it fell within this 1-year period. We continued to follow all subjects who discontinued DMT until their exit in order to examine the patterns of DMT reinitiations. Reinitiations were categorized as within 1 year after discontinuation, between 1 and 2 years after discontinuation, and $\geq 2$ years after discontinuation. In a sensitivity analysis, we modified the persistence definition to allow a gap in therapy of 180 days, increasing our confidence that the medication was truly discontinued.

We also estimated the adherence for those subjects who remained on DMT at 1-, 3-, and 5-year intervals after the index date. Adherence was measured using the proportion of days covered (PDC) - the number of days of DMT supplied during the time period of interest divided by the number of days in the time period. A PDC of $\geq 80 \%$ was considered to be optimal adherence. ${ }^{7,27}$

\section{Statistical analysis}

We estimated the time to discontinuation of any DMT using Kaplan-Meier survival analyses. Differences in the survival time among individual covariates were assessed for statistical significance with log-rank testing, and Sidak's correction was used for multiple comparisons where appropriate..$^{28}$ The 
associations between potential predictors and persistence were estimated using multivariable Cox-proportional hazard models and were reported as adjusted hazard ratios (aHRs) and $95 \%$ confidence intervals. Covariates were selected based on clinical relevance or statistical importance on univariate analysis $(P<0.1)$ and included age at the index date (continuous), sex, residential region at index date (urban vs rural, identified using postal code), and socioeconomic status (SES) using the forward sortation area (the first 3 digits of the postal code) to determine the annual household income (low, medium, and high). We included the year of MS diagnosis (1996-2000, 2001-2005, and 2006-2011), the time from the date of MS diagnosis to the index date, and DMT dispensed at the index date. We also included a measure of "pill burden," estimated by counting the number of unique ATC codes (identified at the 4 th level) in the 1 year before the index date (excluding corticosteroids used for treating MS relapses and DMTs). To assess the effect of hospitalizations on DMT persistence, we used a time-dependent variable describing acute inpatient (overnight) hospitalizations after the index date, but before the discontinuation date. Both MS-specific and non-MS hospitalizations were included, with MSspecific hospitalizations defined as those for which MS was listed as the most responsible diagnosis for the admission. To determine if adherence was a predictor of persistence, we examined all subjects who remained on DMT for at least 1 year after the index date. Multivariable Cox-proportional hazard models included the covariates outlined above, with the addition of an adherence variable. Model assumptions were assessed using standard methods.

\section{Ethics}

Ethics approval for this study was granted by the University of Manitoba Research Ethics Board, and the Health Information Privacy Committee of the Government of Manitoba granted approval to access administrative data. The analyses were conducted using SAS version 9.4 (SAS Institute Inc., Cary, NC, USA).

\section{Results}

A total of 721 subjects met the inclusion criteria and were followed for a median of 7.8 years (interquartile range [IQR] 6.1). The mean age at starting a DMT (index date) was 37.6 (standard deviation 9.8) years and $74.2 \%$ were female. The median duration from the diagnosis date to starting a DMT was 263 (IQR 596) days. Beta-interferon-1b, the first therapy available in Manitoba, was most commonly initiated (33.4\%), followed by subcutaneous beta-interferon-1a (22.9\%), intramuscular beta-interferon-1a $(22.5 \%)$, and glatiramer acetate (21.2\%) (Table 1$)$.

The median time to discontinuation of all first-line DMTs was 4.2 years (25th and 75th percentile: $1.7,10.6$ years) and did not differ by the initial DMT prescribed (Figure 1). After the first year of therapy, $84 \%$ subjects remained on their initial DMT, but this decreased to $50 \%$ after 2 years. Subjects who were older when starting a DMT were less likely to discontinue it than those who were younger (aHR 0.97, 95\% confidence interval [CI]: 0.96-0.98) (Table 2). Lower SES was associated with a higher hazard for discontinuing DMT, but this did not reach statistical significance. Other demographic factors such as sex and region of residence were not associated with DMT discontinuation nor was the number of non-MS medication

Table I Characteristics of study cohort $(n=72 I)$

\begin{tabular}{|c|c|}
\hline Characteristics & \\
\hline Age at MS diagnosis (years), mean (SD) & $36.5(9.7)$ \\
\hline \multicolumn{2}{|l|}{ Year of MS diagnosis, n (\%) } \\
\hline $1996-2000$ & $263(36.5)$ \\
\hline $200 I-2005$ & $296(41.0)$ \\
\hline $2006-2012$ & $162(22.5)$ \\
\hline Age at index date (years), mean (SD) & $37.6(9.8)$ \\
\hline Female, $\mathrm{n}(\%)$ & $535(74.2)$ \\
\hline \multicolumn{2}{|l|}{ Socioeconomic status at index date, $n(\%)$} \\
\hline Low & $244(33.8)$ \\
\hline Medium & $247(34.3)$ \\
\hline High & $230(31.9)$ \\
\hline \multicolumn{2}{|l|}{ Residence, n (\%) } \\
\hline Rural & $298(41.3)$ \\
\hline Urban & $423(58.7)$ \\
\hline \multicolumn{2}{|l|}{ Time to DMT initiation from MS diagnosis (days), $\mathrm{n}(\%)$} \\
\hline$\leq 90$ & $154(2 \mid .4)$ \\
\hline $91-365$ & $280(38.8)$ \\
\hline $366-730$ & $119(16.5)$ \\
\hline$>730$ & $168(23.3)$ \\
\hline \multicolumn{2}{|l|}{ Initial DMT, n (\%) } \\
\hline Beta-interferon-Ia (IM) & $162(22.5)$ \\
\hline Beta-interferon-Ia (SC) & $165(22.9)$ \\
\hline Beta-interferon-Ib & $24 I(33.4)$ \\
\hline Glatiramer acetate & $153(2 \mid .2)$ \\
\hline \multicolumn{2}{|c|}{$\begin{array}{l}\text { Number of non-MS ATC medication classes in I year prior to index } \\
\text { date, } n(\%)\end{array}$} \\
\hline 0 & $76(10.5)$ \\
\hline $1-2$ & $212(29.4)$ \\
\hline $3-4$ & $186(25.8)$ \\
\hline$\geq 5$ & $247(34.3)$ \\
\hline MS-related hospitalization after initiating DMT, n (\%) & $23(3.2)$ \\
\hline Non-MS-related hospitalization after initiating & $78(12.8)$ \\
\hline \multicolumn{2}{|l|}{ DMT, n (\%) } \\
\hline $\begin{array}{l}\text { Follow-up time (index date to study end) (years), } \\
\text { median (IQR) }\end{array}$ & $7.8(6.1)$ \\
\hline
\end{tabular}

Abbreviations: MS, multiple sclerosis; DMT, disease-modifying therapy; ATC, anatomic therapeutic chemical; IM, intramuscular; SC, subcutaneous; SD, standard deviation; IQR, interquartile range. 


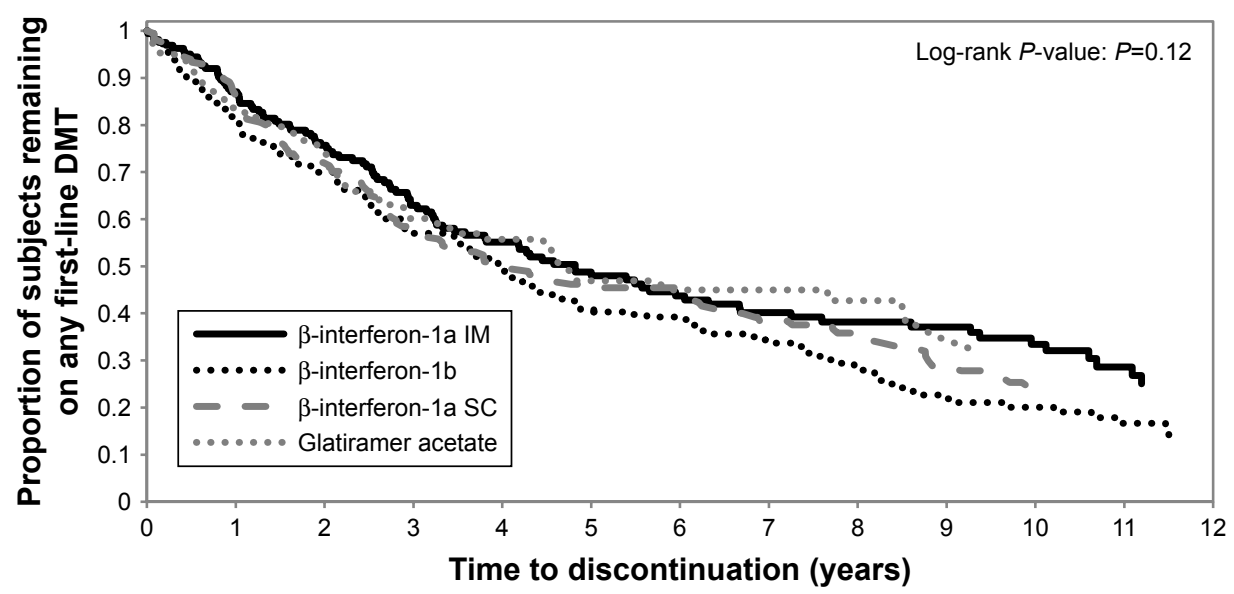

Figure I Time to discontinuation of disease-modifying therapy based on the initial agent prescribed. Abbreviations: $\beta$, beta; IM, intramuscular; SC, subcutaneous; DMT, disease-modifying therapy.

Table 2 Multivariable hazard ratio of time to discontinuation of disease-modifying therapy

\begin{tabular}{|c|c|}
\hline Covariate & $\begin{array}{l}\text { Adjusted hazard } \\
\text { ratio }(95 \% \mathrm{Cl})\end{array}$ \\
\hline Age at index date (years) & $0.97(0.96-0.98)$ \\
\hline \multicolumn{2}{|l|}{ Year of MS diagnosis } \\
\hline 1996-2000 & Reference \\
\hline $200 I-2005$ & $1.57(1.25-1.97)$ \\
\hline $2006-2012$ & $1.46(1.04-2.04)$ \\
\hline \multicolumn{2}{|l|}{ Sex } \\
\hline Female & Reference \\
\hline Male & $1.19(0.95-1.49)$ \\
\hline \multicolumn{2}{|l|}{ Socioeconomic status at index date } \\
\hline High & Reference \\
\hline Medium & I.0I $(0.80-1.28)$ \\
\hline Low & $1.29(0.99-1.68)$ \\
\hline \multicolumn{2}{|l|}{ Residence } \\
\hline Rural & Reference \\
\hline Urban & $1.12(0.90-1.40)$ \\
\hline \multicolumn{2}{|l|}{ Time to DMT initiation from MS diagnosis (days) } \\
\hline$\leq 90$ & Reference \\
\hline $91-365$ & $1.13(0.86-1.48)$ \\
\hline $366-730$ & $1.14(0.82-1.58)$ \\
\hline$>730$ & $1.65(1.19-2.29)$ \\
\hline \multicolumn{2}{|l|}{ Initial DMT } \\
\hline Glatiramer acetate & Reference \\
\hline Interferon-beta-Ia (IM) & $0.94(0.68-1.29)$ \\
\hline Interferon-beta-Ia (SC) & $1.05(0.76-1.44)$ \\
\hline Interferon-beta-Ib & I.3I (0.98-I.75) \\
\hline \multicolumn{2}{|c|}{$\begin{array}{l}\text { Number of non-MS ATC medication classes in I year prior to } \\
\text { index date }\end{array}$} \\
\hline 0 & Reference \\
\hline $1-2$ & $1.09(0.68-1.36)$ \\
\hline $3-4$ & $1.23(0.87-1.75)$ \\
\hline$\geq 5$ & $1.20(0.85-1.68)$ \\
\hline MS-related hospitalization after initiating $\mathrm{DMT}^{\mathrm{a}}$ & $1.40(1.14-1.72)$ \\
\hline Non-MS-related hospitalization after initiating $\mathrm{DMT}^{\mathrm{a}}$ & $1.00(0.83-1.21)$ \\
\hline \multicolumn{2}{|c|}{ 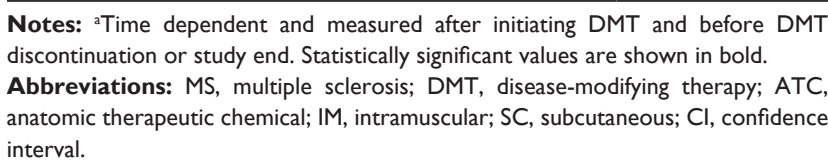 } \\
\hline
\end{tabular}

classes from the year before starting a DMT. A more recent diagnosis of MS and a greater lag time between the MS diagnosis and DMT initiation date were associated with a greater hazard for discontinuing therapy (Table 2). The occurrence of MS-related hospitalizations after starting a DMT was also associated with an increased risk of discontinuing therapy (aHR 1.40, 95\% CI: 1.14-1.72). Findings were similar when a gap of 180 days was used to define persistence.

A total of $125(17.7 \%)$ subjects switched to a different first-line injectable DMT during the study period; 34 (27.2\%) of them switched twice. Of the 451 subjects who discontinued DMT during the study period, 259 (57.4\%) eventually started a new first-line injectable DMT or reinitiated the one they had previously discontinued. Most reinitiations (73.4\%) occurred within the first year of discontinuation; $13.1 \%$ reinitiations occurred between 1 and 2 years, and $13.5 \%$ of subjects restarted a DMT $>2$ years after their initial discontinuation.

Adherence was high in subjects who remained on DMT for at least 1 year, with $80.0 \%$ exhibiting optimal adherence, defined as $\geq 80 \%$, in the first year of therapy. The proportion of adherent subjects increased with the length of time persisting on therapy. Specifically, among those persisting on therapy for 3 years, $83.5 \%$ of subjects demonstrated optimal adherence. Among those persisting on therapy for 5 years, $86.0 \%$ of subjects demonstrated optimal adherence. Subjects with good adherence in the first year of therapy were more likely to remain on DMT compared to those with poor adherence (aHR 0.58, 95\% CI: 0.45-0.75).

\section{Discussion}

In this population-based MS cohort, half of the subjects remained on a first-line injectable DMT for $>4$ years. 
Although $\sim 60 \%$ of the cohort discontinued DMT at some point during the study period, $57.4 \%$ resumed or started a different DMT, with three-quarters of these occurring within 1 year of the discontinuation. These results are similar to a recent study examining the adherence and persistence to the first-line DMTs in three Canadian provinces, including Manitoba. The median time to discontinuation of any DMT for the Manitoba cohort in that study was slightly longer at 4.6 years (95\% CI: 4.1-5.0), but inclusion criteria for the study differed slightly. ${ }^{14}$ Our results differ from a recent study conducted at the Calgary MS Clinic that reported a median time to discontinuation of injectable first-line DMTs of 11.1 years (95\% CI: 10.4-12.0) ${ }^{29}$ and an Italian study that reported a median time to discontinuation of beta-interferon $1 \mathrm{a} / \mathrm{b}$ of 11.98 years (95\% CI: $10.78-13.30) .{ }^{30}$ However, unlike our study which used population-based administrative data based on actual drug dispensations, these studies assessed ongoing DMT use based on participant history provided at clinic reviews. This type of subjective persistence measure is prone to recall bias, and individuals may provide responses that they perceive their neurologist or clinician wants to hear rather than what may be a true representation of their medication utilization. ${ }^{31}$ However, it is more likely that differences in unmeasured patient characteristics, and/or practice variations related to treatment recommendations, support, and patient education between the two sites, are responsible for the differences observed.

Optimal adherence ( $\geq 80 \%$ ) was high in subjects who remained on DMT, and optimal adherence in the first year of DMT use was a strong predictor of whether an individual was going to remain on DMT. Although adherence and persistence are related, they are still two different aspects of medication taking. As clinical outcomes are affected by not only how well individuals take their medications, but also by how long they take them for, ${ }^{32}$ it is important to examine both the aspects when evaluating drug utilization patterns.

Age at the time of DMT initiation was associated with DMT discontinuation; those who were older when starting a DMT had a lower risk of discontinuing their DMT compared with those who started at a younger age. Similar findings were observed in a clinical cohort of MS patients from British Columbia $^{25}$ and a recent study examining adherence and persistence to first-line DMTs in three Canadian provinces. ${ }^{14}$ Our results are also consistent with previous work examining persistence for other chronic medication classes including statins, antihypertensives, bisphosphonates, and oral antidiabetic agents, where the risk for discontinuing drugs declined in a linear fashion with age. ${ }^{33}$
The initial choice of DMT did not influence our results, as none were significantly associated with an increased risk for discontinuing DMT. This is consistent with our previous work that demonstrated no single first-line DMT agent was a predictor of adherence or persistence, ${ }^{14}$ although it differs from other studies that have reported associations between specific DMTs and persistence. ${ }^{26,30,34}$ This variation may be due to differences in methodologies including different data sources and the use of nonpopulation-based cohorts. Among these studies, no single agent has shown a consistent association with persistence, which is not surprising considering that efficacy is similar between all the first-line injectable DMTs, ${ }^{35}$ and that route of administration is also similar. However, as newer therapies emerge with differing efficacy, routes of administrative, and adverse effect profiles, this may change.

Drug cost is commonly recognized as a barrier to medication adherence and persistence for many chronic conditions. ${ }^{6,36,37}$ However, financial reasons are not typically identified as a major predictor for poor adherence or persistence in $\mathrm{MS},{ }^{29,38-40}$ which is initially unexpected considering the first-line DMTs can range from $\$ 16,000$ to $\$ 20,000$ per year in Canada. Our results support this, as SES was not associated with DMT discontinuations. One reason for this may be that almost all individuals on a DMT in Canada receive some coverage through provincial drug plans and/or third-party insurance.

The year of MS diagnosis was associated with DMT discontinuation; those subjects who were diagnosed more recently were more likely to discontinue their DMT compared to those diagnosed between 1996 and 2000. This is consistent with previous Canadian studies. ${ }^{14,25,29}$ We suggest that this may be due to the availability of DMTs. The first DMT for MS was approved for use in Canada in 1995, and until this time, the only treatment for MS was high-dose corticosteroids to treat relapses. As more DMTs became (and continue to become) available, individuals may be less willing to remain on a DMT, especially if they are experiencing adverse effects. The shift to earlier diagnosis and treatment of MS may also play a role, as individuals initiating DMTs more recently may have less severe disease compared to those who were treated when DMTs first became available. Less severe disease may result in a perceived lack of benefit of, or need for, DMT, which has been shown to be a main predictor of DMT discontinuation in MS. ${ }^{38-40}$

We observed that the subjects who waited longer after their MS diagnosis to start a DMT were more likely to discontinue when compared to those who started a DMT within 3 months of their diagnosis, although statistical significance 
was only achieved in those waiting $>2$ years. There are several potential reasons for this. First, those individuals who started DMT shortly after the MS diagnosis may have had a more severe presentation of the disease. As disease severity or perceived disease "threat" has been shown to positively correlate with medication adherence, ${ }^{41}$ these individuals may have been more motivated to remain on DMT. Individuals who waited to initiate DMT may have had less severe disease, or may have been less convinced about the need or benefit of the DMT, and therefore may be more likely to discontinue therapy once it had been started.

Acute (overnight) hospitalizations occurred in 16\% of our cohort during the study period; $3 \%$ of hospitalizations were MS related. Previous research has shown the three most common non-MS reasons for hospitalizations among the MS population in Manitoba are diseases of the digestive, genitourinary, and circulatory systems. ${ }^{42}$ We observed an association between MS-related hospitalizations that occurred while taking DMT and the risk for discontinuing DMT. The same association was not seen when examining non-MS-related hospitalizations. This suggests that the hospitalization was likely due to an MS relapse or disease progression, or a potential adverse reaction to the DMT, any of which may have resulted in a change or discontinuation in therapy.

Over $60 \%$ of our cohort discontinued DMT at some point during the study period. However, it is important to note that $57 \%$ of these individuals eventually resumed their initial DMT or started a different DMT, with the majority occurring within 1 year of discontinuation. Our findings are similar to results from a recent study at the Calgary MS Clinic which reported $54 \%$ of individuals restarted a DMT after an initial discontinuation..$^{29}$ These temporary disruptions in therapy may have been due to monitoring recommendations for withholding doses for typically transient issues like liver enzymes or hematologic abnormalities. Although there is no consensus on how long an individual with MS should be treated with a DMT, ${ }^{43,44}$ there is a general agreement that most individuals need to remain on DMT long term to receive benefits. There is currently a paucity of clinical trial data on what happens to individuals when they discontinue DMT. However, recent preliminary evidence from observational studies suggest increased relapses and disability in those who discontinue DMT. ${ }^{45,46}$ Therefore, the fact that many subjects in our cohort restarted a DMT is reassuring.

A major strength of our study was the ability to examine DMT persistence and adherence over a 15 -year period in a large, population-based cohort of individuals with MS. However, there are still limitations that should be considered. Inherent in all observational studies is the lack of clinical information which could influence our results, such as disease severity, disease progression, and comorbidity. As there is no validated or consistent measure for MS severity or comorbidity burden when using administrative data, we adjusted for prior hospitalizations and non-DMT medication use as proxy measures. Although we did account for DMT discontinuation due to pregnancy, we were not able to determine other motives for DMT discontinuation, some of which may have been for legitimate reasons such as adverse effects or disease progression. Because we estimated adherence using administrative data, we made the assumption that the dispensed medication was actually administered, which may not have always been the case. However, adherence measures that rely on direct observation or "pill counts" would not have been feasible. Finally, due to small numbers, we were unable to include the available second-line DMTs (natalizumab and fingolimod) in our analyses of persistence and adherence.

Our study examined the persistence to the first-line injectable DMT between 1996 and 2011. The emergence of new DMTs in the last few years has increased the treatment options for MS. The increased efficacy and more favourable dosing regimens of these newer agents provide an opportunity for patients to escalate to second-line treatment sooner, rather than switching between the first-line agents as was common in the past. However, in Canada, the firstline injectable DMTs are still initiated in many patients, likely due to their longevity and established safety profile, and switches between these therapies may still occur due to safety considerations or the thresholds which need to be met to qualify for access to second-line treatments. As such, these results still provide clinicians with relevant information for managing their patients on these medications. As we gain more long-term data with the newer agents, and additional therapies continue to emerge, factors associated with persistence may change.

\section{Conclusion}

We found that over half of the individuals receiving a first-line injectable DMT for MS in Manitoba remain on therapy for at least 4 years. While DMT discontinuation did occur in $60 \%$ of the cohort, the majority end up resuming or reinitiating a new DMT within 1 year of the discontinuation. Although the optimal length of DMT for MS is yet to be established, these 
numbers are encouraging. We also found factors associated with an increased risk for discontinuing DMT, including a younger age, increased time from MS diagnosis until DMT initiation, and prior MS-related hospitalizations. Although not all of these factors are modifiable, they may help practitioners enhance MS care by identifying individuals who may be at particular risk for DMT discontinuation.

\section{Acknowledgment}

The results and conclusions are those of the authors, and no official endorsement to Manitoba Health, Seniors and Active Living or other data providers is intended or should be inferred.

\section{Disclosure}

Dr Marrie received research funding from Canadian Institutes of Health Research, Research Manitoba, Multiple Sclerosis Society of Canada, Multiple Sclerosis Scientific Foundation, National Multiple Sclerosis Society, Rx \& D Health Research Foundation and has conducted clinical trials funded by Sanofi-Aventis. The other authors report no conflicts of interest in this work.

\section{References}

1. Jacobs L, Cookfair D, Rudick R. Intramuscular interferon beta-1a for disease progression in relapsing multiple sclerosis. The Multiple Sclerosis Collaborative Research Group (MSCRG). Ann Neurol. 1996;39:285-294.

2. PRISMS (Prevention of Relapses and Disability by Interferon beta-1a Subcutaneously in Multiple Sclerosis) Study Group. Randomised double-blind placebo-controlled study of interferon beta-1a in relapsingremitting multiple sclerosis. Lancet. 1998;352:1498-1504.

3. Filippini G, Munari L, Incorvaia B, et al. Interferons in relapsing remitting multiple sclerosis: a systematic review. Lancet. 2003;361: 545-552.

4. Cramer JA, Roy A, Burrell A, et al. Medication compliance and persistence: terminology and definitions. Value Health. 2008;11:44-47.

5. Menzin J, Caon C, Nichols C, White LA, Friedman M, Pill M. Narrative review of the literature on adherence to disease-modifying therapies among patients with mutiple sclerosis. JManag Care Pharm. 2013;19(1-a):S24-S40.

6. World Health Organization. Adherence to long-term therapies evidence for action; 2003. Available from: http://www.who.int/ chp/knowledge/publications/adherence_introduction.pdf. Accessed September 1, 2009.

7. Osterberg L, Blaschke T. Adherence to medication. New Engl J Med. 2005;353:487-497.

8. Simpson S, Eurich D, Majumdar S, et al. A meta-analysis of the association between adherence to drug therapy and mortality. BMJ. 2006;333(7557):15.

9. Sokol M, McGuigan K, Vebrugge R, Epstein R. Impact of medication adherence on hospitalization risk and healthcare costs. Med Care. 2005;43:521-530.

10. Tan H, Cai Q, Agarwal S, Stephenson J, Kamat S. Impact of adherence to disease-modifying therapies on clinical and economic outcomes among patients with multiple sclerosis. Adv Ther. 2011;28:51-61.
11. Steinberg S, Faris R, Chang C, Chan A, Tankersley M. Impact of adherence to interferons in the treatment of multiple sclerosis a non-experimental, retrospective, cohort study. Clin Drug Investig. 2010;30:89-100.

12. Ivanova J, Bergman R, Birnbuam H, Phillips A, Stewart M, Meletiche D. Impact of medication adherence to disease-modifying drugs on severe relapse, and direct and indirect costs among employees with multiple sclerosis in the US. J Med Econ. 2012;15:601-609.

13. Hansen K, Schussel K, Kieble M, et al. Adherence to disease modifying drugs among patients with multiple sclerosis in Germany: a retrospective cohort study. PLoS One. 2015;10(7):e0133279.

14. Evans C, Marrie RA, Zhu F, et al. Adherence and persistence to drug therapies for multiple sclerosis: a population-based study. Mult Scler Relat Disord. 2016;8:78-85.

15. Milanese C, La Mantia L, Palumbo R, et al. A post-marketing study on interferon beta $1 \mathrm{~b}$ and $1 \mathrm{a}$ treatment in relapsing-remitting multiple sclerosis: different response in drop-outs and treated patients. $J$ Neurol Neurosurg Psychiatry. 2003;74:1689-1692.

16. Portaccio E, Zipoli V, Siracusa G, Sorbi S, Amato M. Long-term adherence to interferon B therapy in relapsing-remitting multiple sclerosis. Eur Neurol. 2007;59:131-135.

17. Lafata J, Cerghet M, Dobie E, et al. Measuring adherence and persistence to disease-modifying agents among patients with relapsing remitting multiple sclerosis. J Am Pharm Assoc. 2008;48: $752-757$.

18. Daugherty K, Butler J, Mattingly M, Ryan M. Factors leading patients to discontinue multiple sclerosis therapies. J Am Pharm Assoc. 2005;45: 371-375.

19. O'Rourke K, Hutchinson M. Stopping beta-interferon therapy in multiple sclerosis: an analysis of stopping patterns. Mult Scler. 2005; 11:46-50.

20. Klauer T, Zettl U. Compliance, adherence, and the treatment of multiple sclerosis. J Neurol. 2008;255 (Suppl 6):87-92.

21. Tremlett H, Oger J. Interrupted therapy - stopping and switching of the B-interferons prescribed for MS. Neurology. 2003;61:551-554.

22. Clerico M, Barbero P, Contessa G, Ferrero C, Durelli L. Adherence to interferon-beta treatment and results of therapy switching. J Neurol Sci. 2007;259:104-108.

23. Health Information Managment Branch. Population Report. Winnipeg, MB: Manitoba Health and Healthy Living; 2008.

24. Marrie R, Yu N, Blanchard J, Leung S, Elliot L. The rising prevalence and changing age distribution of multiple sclerosis in Manitoba. Neurology. 2010;74:465-471.

25. Evans C, Tam J, Kingwell E, Oger J, The UBC Neurologists, Tremlett H. Long-term persistence with the immunomodulatory drugs for multiple sclerosis: a retrospective database study. Clin Ther. 2012;34:341-350.

26. Reynolds M, Stephen R, Seaman C, Rajagopalan K. Persistence and adherence to disease modifying drugs among patients with multiple sclerosis. Curr Med Res Opin. 2010;26:663-674.

27. Karve S, Cleves M, Helm M, Hudson T, West D, Martin B. Good and poor adherence: optimal cut-point for adherence measures using administrative claims data. Curr Med Res Opin. 2009;25: 2303-2310.

28. Sidak Z. Rectangular confidence regions for the means of multivariate normal distributions. J Am Statist Assoc. 1967;62:626-633.

29. Zhornitsky S, Greenfield J, Koch M, et al. Long-term persistence with injectable therapy in relasping-remitting multiple sclerosis: an 18-year observational cohort study. PLoS One. 2015;10(4):e0123824.

30. Moccia M, Palladino R, Carotenuto A, et al. Predictors of long-term interferon discontinuation in newly diagnosed relapsing multiple sclerosis. Mult Scler Relat Disord. 2016;10:90-96.

31. Nguyen T-M-U, La Caze A, Cottrell N. What are validated self-report adherence scales really measuring?: a systematic review. $\mathrm{Br} \mathrm{J}$ Clin Pharmacol. 2013;77:427-445. 
32. Cramer J, Roy A, Burrell A, Fairchild C, Fuldeore M, Ollendorf D. Medication compliance and persistence: terminology and definitions. Value Health. 2008;11:44-47.

33. Yeaw J, Benner J, Walt J, Sian S, Smith D. Comparing adherence and persistence across 6 chronic medication classes. J Manag Care Pharm. 2009;15:728-740.

34. Correia I, Marques IB, Sousa M, et al. Predictors of first-line treatment persistence in a Portuguese cohort of relapsing-remitting multiple sclerosis. J Clin Neurosci. 2016;33:73-78.

35. La Mantia L, Di Pietrantonj C, Rovaris M, et al. Interferons-beta versus glatiramer acetate for relapsing-remitting multiple sclerosis. Cochrane Database Syst Rev. 2016;(11):CD009333.

36. McHorney C, Spain C. Frequency of and reasons for medication nonfulfillment and non-persistence among American adults with chronic disease in 2008. Health Expect. 2010;14:307-320.

37. Brown M, Bussell J. Medication adherence: WHO cares? Mayo Clin Proc. 2011;86:304-314.

38. Treadaway K, Cutler G, Salter A, Lynch S, Simsarian J, Corboy J. Factors that influence adherence with disease-modifying therapy in MS. J Neurol. 2009;256:568-576.

39. Devonshire V, Lapierre Y, Macdonell R, et al. The Global Adherence Project (GAP): a multicenter observational study on adherence to disease-modifying therapies in patients with relasping-remitting multiple sclerosis. Eur J Neurol. 2011;18:69-77.

40. Fox R, Salter A, Tyry T, et al. Treatment discontinuation and disease progression with injectable disease-modifying therapies - Findings from the North American Research Committee on Multiple Sclerosis Database. Int J MS Care. 2013;15:194-201.
41. DiMatteo MR, Haskard K, Williams S. Health beliefs, disease severity, and patient adherence: a meta-analysis. Med Care. 2007;45:521-528.

42. Marrie RA, Elliot L, Marriott J, et al. Dramatically changing rates and reasons for hospitalization in multiple sclerosis. Neurology. 2014; 83:929-937.

43. Butler M, Forte M, Schwehr N, Carpenter A, Kane R. Decisional Dilemmas in Discontinuing Prolonged Disease-Modifying Treatment for Multiple Sclerosis. Minnesota Evidence-based Practice Centre: Agency for Healthcare Research and Quality; 2015.

44. Freedman M, Selchen D, Arnold D, et al. Treatment optimization in MS: Canadian MS Working Group Updated Recommendations. Can J Neurol Sci. 2013;40:307-323.

45. Kister I, Spelman T, Duquette P, et al. Doctor, can I stop my medicine? Analysis of disease course after stopping disease-modifying therapy in stable MS patients (P5.192). Neurology. 2015;84: Supplement P5.192.

46. Bonenfant J, Bajeux E, Deburghgraeve V, Le Page E, Edan G, Kerbrat A. Can we stop immunomodulatory treatments in secondary progressive multiple sclerosis? Eur J Neurol. 2017;24:237-244.

47. Quail J, Lix L, Osman BA, Teare G. Comparing comorbidity measures for predicting mortality and hospitalization in three population-based cohorts. BMC Health Serv Res. 2011;11:146. 


\section{Supplementary material}

Table SI ICD codes for demyelinating diseases of the central nervous system used for determining the date of multiple sclerosis diagnosis

\begin{tabular}{ll}
\hline Claim & ICD-9/ICD- I 0 codes \\
\hline Optic neuritis & $377.3 / \mathrm{H} 46$ \\
Acute transverse myelitis & $323.82 / \mathrm{G} 37$ \\
Acute disseminated encephalomyelitis & $323 / \mathrm{G} 36.9$ \\
Demyelinating disease of CNS unspecified & $341.9 / \mathrm{G} 37.8$ \\
Other acute disseminated demyelination & $\mathrm{G} 36$ \\
Multiple sclerosis & $340 / \mathrm{G} 35$ \\
Neuromyelitis optica & $341.0 / \mathrm{G} 36.0$ \\
\hline
\end{tabular}

Abbreviations: ICD, International Classification of Diseases; CNS, central nervous system.

\section{Publish your work in this journal}

Patient Preference and Adherence is an international, peer-reviewed, open access journal that focuses on the growing importance of patient preference and adherence throughout the therapeutic continuum. Patient satisfaction, acceptability, quality of life, compliance, persistence and their role in developing new therapeutic modalities and compounds to optimize clinical outcomes for existing disease states are major areas of interest for the journal. This journal has been accepted for indexing on PubMed Central. The manuscript management system is completely online and includes a very quick and fair peer-review system, which is all easy to use. Visit http://www. dovepress.com/testimonials.php to read real quotes from published authors.

\footnotetext{
Submit your manuscript here: http://www.dovepress.com/patient-preference-and-adherence-journal
} 